\title{
Abundance variation of ungulates in two protected areas of the Colombian Guayana estimated with occupancy models
}

\author{
Bibiana Gómez $^{1,2 *}$, Olga Montenegro ${ }^{1,3}$ y Pedro Sánchez-Palomino ${ }^{1,4}$
}

\author{
1 Grupo Conservación y Manejo de Vida Silvestre, Instituto de Ciencias Naturales, Universidad Nacional de Colombia. Carrera 30 \# \\ 45 - 03. Bogotá, Colombia. \\ ${ }^{2}$ Estudiante de Doctorado en Ciencias Biológicas, GESEAA-Universidad de Buenos Aires. Buenos Aires, Argentina. E-mail: \\ bgomezv@gmail.com (BG). \\ ${ }_{3}^{3}$ Profesora Asociada, Instituto de Ciencias Naturales, Universidad Nacional de Colombia. Carrera 30 \# 45 - 03, Edificio 425, oficina \\ 108, Bogotá-Colombia. E-mail: olmontenegrod@unal.edu.co (OM). \\ ${ }^{4}$ Profesor Asistente, Departamento de Biología, Universidad Nacional de Colombia. Carrera 30 \# 45 - 03, Edificio 421, oficina $120 \mathrm{~B}$. \\ Bogotá, Colombia. E-mail: psanchezp@unal.edu.co (PS-P). \\ ${ }^{*}$ Corresponding author
}

Six ungulate species inhabit the Colombian Guiana Shield, a patchily-distributed biogeographic region characterized by poor nutrient soils and by harboring the only forest in Colombia growing on a Precambrian surface. In Colombia, two protected areas are located on the Colombian Guiana Shield, the Tuparro National Natural Park (PNNT, for its Spanish initials) and the Puinawai National Natural Reserve (RNNP, for its Spanish initials), which have a large difference in the configuration and availability of native forest. These ungulates are three deer species (Odocoileus virginianus, Mazama americana and M. nemorivaga), two peccary species (Pecari tajacu and Tayassu pecari) and one tapir specie (Tapirus terrestris). The aims of this study were to estimate and compare the abundance of those six ungulate species in two protected areas located within the Guiana Shield region in Colombia and analyze the relationship with the landscape forest amount and distance to human settlements. The study sites were in PNNT where the landscape is dominated by natural savannas mixed with riparian forests, and RNNP covered by tropical forests. We estimated the relative abundance of each ungulate species using repeated presence-absence data with the occupancy model with heterogeneous detection of Royle and Nichols (2003). Presence detection was obtained from both direct observations and tracks. The direct sightings and tracks we sampled along six transects in PNNT and nine in RNNP ( $244.4 \mathrm{~km}$ at PNNT and $257 \mathrm{~km}$ at RNNP) and track plots of $0.25 \mathrm{~m}^{2}$ ( 211 at PNNT and 297 at RNNP). The relative abundance of tapir, collared peccary (P. tajacu) and brocket deer (Mazama spp.) were different between protected areas; we found no differences in the white-lipped peccary abundance (T. pecari). Relative abundance estimation was higher at RNNP for tapirs and brocket deers. In contrast, collared peccary was more abundant in the PNNT. There was positive effect of floodplain forest area abundance of white-lipped peccary. The distance to human settlements had positive effect on collared peccary abundance and negative effect on tapir and white tailed deer (O. virginianus). The higher abundance in the RNNP for tapirs and Mazama spp., brocket deer was unrelated to the forest area, so the difference may be related to local habitat conditions. The white-lipped peccary is sensitive to the forest area in the landscape, but has also been found that riparian forests act as natural corridors. Failure to find differences in abundance can be an indicator of the high habitat quality for these species. The collared peccary has been positively associated to a moderate degree of subdivision of forest at landscape (number of patchs), which could explain the higher abundance in the PNNT. The positive relationship of white-lipped peccary with the floodplain forest coincides with the patterns observed in other Amazonian areas. Finally, the negative effect of the distance from settlements for brocket deer and tapir may be related to low hunting pressure on these species, contrary to what was found for the collared peccary. The six ungulate species show low detectability, which is associated with low abundances.

Seis especies de ungulados habitan la región de la Guayana colombiana, una región biogeográfica de distribución discontinua caracterizada por tener suelos muy antiguos, pobres en nutrientes y albergar los únicos bosques que crecen en una superficie de origen precámbrico. En colombia dos áreas protegidas están ubicadas en el escudo guayanés, el Parque Natural Nacional Tuparro (PNNT) y la Reserva Nacional Natural Puinawai (RNNP), las cuales presentan una gran diferencia en la configuración y disponibilidad natural del bosque. Los ungulados corresponden a tres especies de venado (Odocoileus virginianus, Mazama americana y M. nemorivaga), dos especies de pecaríes (Pecari tajacu y Tayassu pecari) y una especie de tapir (Tapirus terrestris). El objetivo de este estudio fue estimar y comparar la abundancia relativa de estas seis especies en dos áreas protegidas de la guayana colombiana y analizar su relación con la cantidad de bosque en el paisaje y la distancia a poblados humanos. El estudio se realizó en PNNT donde predominan sabanas naturales mezcladas con bosques ribereños y la RNNP donde predomina el bosque en un continuo. Se estimó la abundancia relativa con el modelo de ocupación con heterogéneidad en la detección de Royle y Nichols (2003). Las detecciones se obtuvieron de observación directa e indirecta a lo largo de 6 senderos en el PNNT ( $228.5 \mathrm{~km}$ en PNNT) y 9 en RNNP ( $298.8 \mathrm{~km}$ en RNNP) y en parcelas de $0.25 \mathrm{~m}^{2}$ (211 en PNNT y 297 en RNNP). La abundancia relativa (AR) del tapir, el pecarí de collar y los venados Mazama spp. varió entre las áreas protegidas. En contraste, no encontramos diferencia para el pecarí de labios blancos. La AR fue mayor en la RNNP para el tapir y Mazama spp., mientras que para el pecarí de collar fue mayor en el PNNT. El porcentaje de bosque inundable tuvo efecto positivo en la AR del 
pecarí de labios blancos. La distancia a poblados tuvo un efecto positivo en la AR del pecarí de collar y un efecto negativo para el tapir y el venado cola blanca. La mayor abundancia en la RNNP para los tapires y los venados Mazama spp., no tuvo relación con la cantidad de bosque en el paisaje y las diferencias podrían estar relacionadas a condiciones del hábitat a escala local. El pecarí de labios blancos es una especie sensible a la cantidad de bosque en el paisaje, pero también se ha encontrado que los bosques ribereños funcionan como corredores naturales. El no encontrar diferencia en la abundancia puede ser un indicador del buen estado de estas áreas para la especie. El pecarí de collar se ha encontrado asociado de manera positiva a un grado moderado de división del bosque en parches, lo que podría explicar la mayor abundancia en el PNNT. La relación positiva del pecarí de labios blancos con el bosque inundable coincide con los patrones observados en otras áreas de la Amazonía. Por último, el efecto negativo de la distancia a poblados para el venado cola blanca y el tapir puede estar relacionado con baja presión de cacería sobre estas especies, contrario a lo encontrado para el pecarí de collar. Las seis especies de ungulados muestran una baja detectabilidad lo que está asociado con sus bajas abundancias.

Key words: Mazama, Odocoileus virginianus, Pecari tajacu, Puinawai, Royle and Nichols model, Tapirus terrestris, Tayassu pecari, Tuparro.

(c) 2016 Asociación Mexicana de Mastozoología, www.mastozoologiamexicana.org

\section{Introducción}

Los ungulados desempeñan un rol fundamental en la dinámica de los bosques neotropicales a través de los procesos de herbivoría, dispersión y depredación de semillas, los cuales tienen influencia en la composición y estructura de la vegetación (Kurten 2013). También son las principales presas de los grandes carnívoros (Ray et al. 2013) y son ampliamente utilizados por las comunidades rurales e indígenas, representando una fuente importante de proteína animal (Peres y Palacios 2007). En muchas localidades la sobreexplotación de los ungulados, en conjunto con la pérdida y la degradación del hábitat han llevado a la disminución de sus poblaciones (Wilkie et al. 2011; Altrichter et al. 2012; Dirzo et al. 2014). Como resultado, varias especies de ungulados se consideran amenazadas de extinción de acuerdo con los criterios de la UICN. Por ejemplo, las especies Tapirus terrestris y Tayassu pecari están en la categoría vulnerable-VU a nivel global (Naveda et al. 2008; Keuroghlian et al. 2013). De otras especies, ni siquiera se conoce su estado de conservación, tal como ocurre con el venado colorado, Mazama americana, la cual se encuentra categorizada con datos insuficientes-DD a nivel global (Duarte y Vogliotti 2015).

La pérdida de los grandes ungulados puede tener efecto a largo plazo sobre la demografía y la densidad de plántulas, lo que podría modificar la composición y la diversidad de la comunidad de árboles de dosel (Beck et al. 2013; Harrison et al. 2013). De igual manera, la extinción de estas especies compromete la seguridad alimentaria de algunas comunidades humanas que dependen de ellas como fuente principal de proteína (Tafur 2010; Martinez-Salas et al. 2016).

Los ungulados presentan una gran variación respecto a la preferencia y uso de coberturas naturales (Bodmer 1990; Desbiez et al. 2009). Las dos especies de venados, M. americana y M. nemorivaga, se encuentran en una variedad de tipos de vegetación a lo largo de su amplia distribución (Weber y Gonzalez 2003). La ocupación de estas dos especies de Mazama en los mismos hábitats es posible a través de evitar la competencia mediante las diferencias en los patrones de actividad (Ferreguetti et al. 2015). Varios estudios indican que M. americana y M. nemorivaga prefieren áreas con bosque de tierra firme (Bodmer 1990; Tobler et al. 2009). El pecarí de collar es una especie generalista de hábitat (Sowls 1997) y tolera un gran rango de condiciones (Altrichter y Boaglio 2004; Reyna-Hurtado y Tanner 2007). Sin embargo prefiere los bosques bajos inundables en lugares con cacería (Reyna-Hurtado y Tanner 2005) 
y los bosques de tierra firme en áreas sin cacería; es más abundante en sitios con baja densidad humana y alejados a las ciudades (Gabor y Hellgren 2000; Gabor et al. 2001). El pecarí de labios blancos usa ampliamente los bosques de tierra firme y los bosques inundables, los cuales prefiere durante la época de inundación (Bodmer 1990; Tobler et al. 2009). Por su parte, el tapir de tierras bajas tampoco se ve afectado por la inundación de los bosques, ya que usa tanto el bosque inundadable como el de tierra firme, aunque muestra preferencia por el primero (Bodmer 1990; Salas 1996; Tobler et al. 2009). De igual forma, aunque el tapir puede estar presente o usar varios tipos de cobertura, siempre muestra tener como hábitat óptimo el bosque continuo (Desbiez et al. 2009) y el uso de hábitat se incrementa con el incremento en la protección contra la cacería (Cruz et al. 2014).

Respecto a la cacería, algunas especies pueden ser más susceptibles que otras a la presión de caza (Reyna-Hurtado y Tanner 2007) y con frecuencia la densidad de ungulados es mucho más baja en áreas sometidas a esta presión (Peres 1996; Naranjo y Bodmer 2007; Peres y Palacios 2007). En otros casos, son las condiciones del hábitat las que mantienen bajas las densidades naturales de ungulados. Esto puede ocurrir por ejemplo en áreas protegidas localizadas en el escudo guayanés caracterizado por tener suelos pobres en nutrientes (Forget y Hammond 2005). En Suramérica, esta región se localiza al norte del continente y se constituye en una región única por sus características geológicas antiguas (Hammond 2005). La región de la Guayana en general enfrenta varias amenazas como la acelerada pérdida y degradación del hábitat y la actividad minera ilegal (de Thoisy et al. 2010), factores que con seguridad afectan las poblaciones de ungulados que aún persisten allí.

En Colombia, la región biogeográfica de la Guayana aflora de forma discontinua a manera de islas en el oriente del país. En esta región biogeográfica se encuentran seis especies de ungulados: el venado colorado (Mazama americana), el venado pardo ( $M$. nemorivaga), el venado cola blanca (Odocoileus virginianus), el pecarí de collar (Pecari tajacu), el pecarí de labios blancos (Tayassu pecari) y el tapir de tierras bajas (Tapirus terrestris) (Patterson y Costa 2012; Richard-Hansen et al. 2015). El estado de conservación de estas especies en la región guayanesa colombiana se desconoce, y solo existen algunas estimaciones de abundancia para pecaríes (Gómez y Montenegro 2012).

Para cualquier estrategia de manejo y conservación de las especies de ungulados es indispensable conocer la abundancia de sus poblaciones y su variación en diferentes tipos de hábitat o a lo largo del tiempo (Morellet et al. 2007). Uno de los métodos más utilizados para la estimación de la abundancia de ungulados en el Neotrópico es el conteo de avistamientos directos de los animales a lo largo de transectos lineales (Burnham et al. 1980). Se ha evaluado incluso la magnitud de los esfuerzos mínimos de muestreo para obtener estimaciones confiables con este método (de Thoisy et al. 2008). Sin embargo, en áreas con bajas densidades de ungulados, el uso de transectos lineales puede no ser suficiente para una estimación confiable (Munari et al. 2011). Por lo tanto, es necesario utilizar otros métodos para tener estimaciones de abundancia en áreas con bajas densidades. Existen algunas alternativas como la propuesta de Royle y Nichols (2003) para obtener estimados de abundancia derivados de un modelo ocupación con heterogeneidad en la detección a partir de medidas repetidas de presencia-ausencia. Los modelos de ocupación se han sugerido para aquellas circunstancias en las cuales no es factible obtener buenos estimados de densidad y se ha propuesto como un sustituto de la abundancia (Royle y Nichols 2003; MacKenzie y Nichols 2004). 
El objetivo del presente trabajo fue estimar y comparar entre dos áreas protegidas la abundancia relativa de seis especies de ungulados, utilizando el modelo de ocupación con heterogeneidad en la detección de Royle y Nichols (2003). Las dos áreas protegidas se ubican en el escudo guayanés colombiano y presentan una gran diferencia en la configuración y disponibilidad natural del bosque que albergan. Una de ellas, la Reserva Nacional Natural Puinawai (RNNP) está dominada por bosques en una disposición continua mientras que la segunda, el Parque Nacional Natural el Tuparro (PNNT) presenta bosques dispuestos en parches naturales inmersos en sabanas tropicales lo que genera un paisaje heterogéneo (Romero et al. 2004). En este trabajo se buscó estimar y comparar la abundancia de los ungulados entre las dos áreas protegidas y el efecto de la cantidad de bosque en el paisaje y la distancia a poblados sobre las estimaciones de abundancia.

\section{Materiales y métodos}

Área de estudio. Este trabajo se realizó en dos áreas protegidas: el Parque Nacional Natural el Tuparro (PNNT) y la Reserva Nacional Natural Puinawai (RNNP). El PNNT está dominado por sabanas de gramíneas mezcladas con bosque húmedo tropical (bosques riparios y en parches naturales), mientras que en la RNNP existe un mosaico de formaciones de bosque continuo (bosques de tierra firme, inundables y caatingas) (Gómez 2010; Figura. 1). Las dos áreas protegidas forman parte del Sistema Nacional de Áreas Protegidas y se encuentran sobre afloramientos del escudo Guayanés en Colombia (Etter 2001).

EI PNNT está localizado en el departamento del Vichada (Figura 1), entre $5^{\circ} 0^{\prime}$ y $5^{\circ} 34^{\prime} \mathrm{N}$ y $-67^{\circ} 50^{\prime}$ y $-69^{\circ} 11^{\prime} \mathrm{W}$, posee $548,000 \mathrm{ha}$, altitudes entre 100 y $330 \mathrm{~m}$ y temperatura media de $27.4^{\circ} \mathrm{C}$ (UAESPNN 1998). El clima es cálido húmedo, posee un régimen de lluvia monomodal y una marcada estacionalidad, con una precipitación promedio anual de 2,366 mm, con un periodo máximo de lluvias entre abril y octubre y uno mínimo entre noviembre y marzo-abril. Esta área protegida posee cinco de los doce ecosistemas de la ecorregión Llanos Orientales, tales como bosques densos de galería y morichales, sabanas de altillanura muy disectadas, sabanas de altillanura plana, sabanas de altillanura arenosa guayanesa y sabanas hiperestacionales (Romero et al. 2004; Correa et al. 2006).

En cuanto a la vegetación en la zona de muestreo del PNNT, diez familias de plantas con flores concentran cerca del $70 \%$ de la riqueza de especies, entre las que predominan la familia Fabaceae (24 especies), Sapotaceae (15), Moraceae (13), Annonaceae (13), Chrysobalanaceae (12) y Burseraceae (12). Las especies con mayor valor de importancia son Oenocarpus bataua, Pouteria sp., Oenocarpus bacaba y Geissospermum reticulatum. Las familias con mayor importancia son Fabaceae, Sapotaceae y Arecaceae (Gómez 2010). En el PNNT, el estudio se localizó en el sector nororiental del parque. Las comunidades indígenas del área de influencia del PNNT pertenecen a la etnia Curripaco con las comunidades de Churuata, Peniel y Pedro Camejo (140 personas, 21 familias) ubicadas en las islas del Raudal de Maipures (Martinez-Salas et al. 2016).

El otro sitio de estudio fue la RNNP, la cual está ubicada en el departamento de Guainía, municipio de Puerto Inírida, y se localiza entre $1^{\circ} 43^{\prime}$ y $2^{\circ} 58^{\prime} \mathrm{N}$ y $-68^{\circ} 41^{\prime}$ y $-69^{\circ}$ $55^{\prime} \mathrm{W}$ (Figura 1). Tiene una extensión de 1,092,500 ha, comprende alturas entre 100 y $500 \mathrm{~m}$ y una temperatura media de $26.6^{\circ} \mathrm{C}$ (UAESPNN 1998). El clima es tropical, 

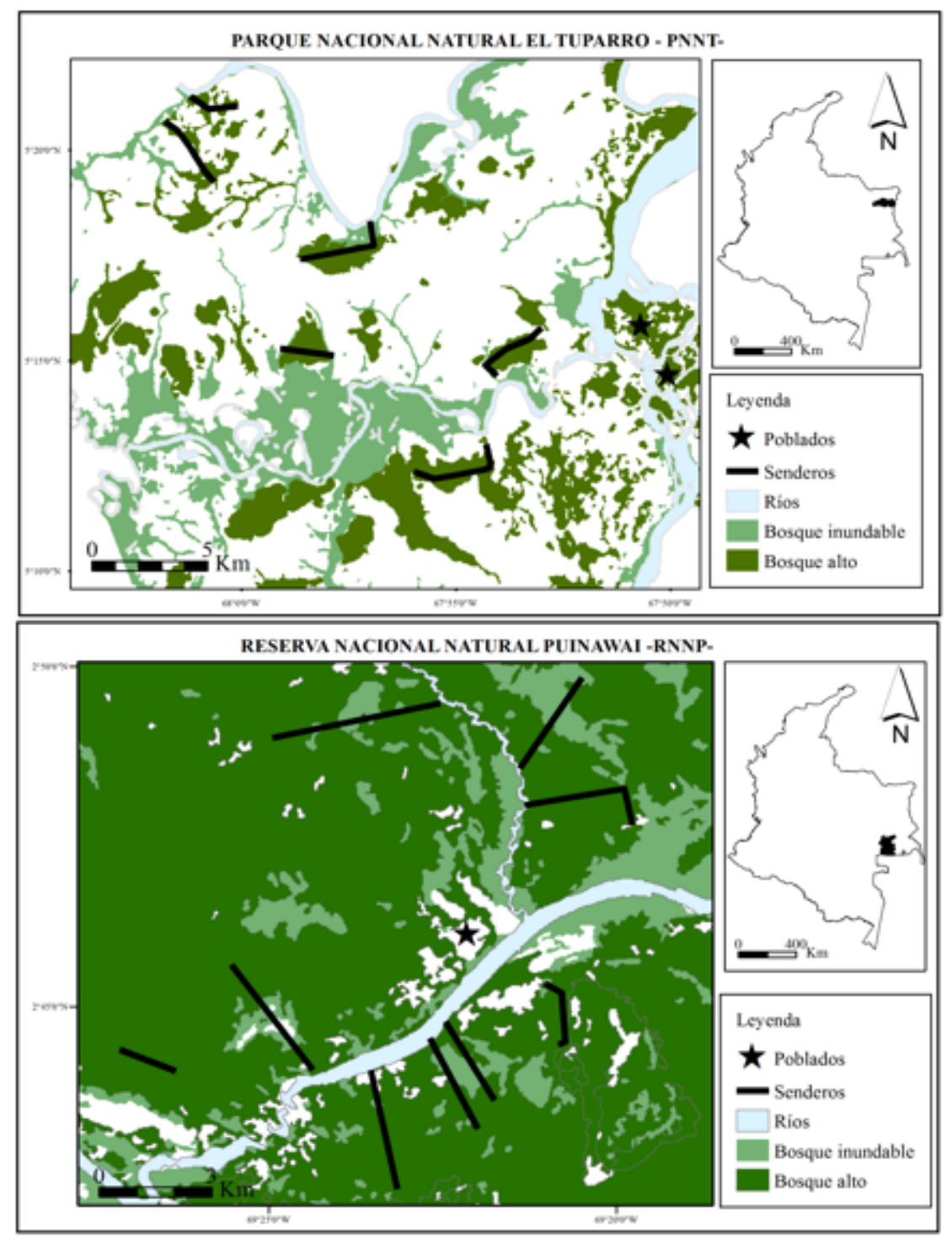

Figura 1. Área de estudio y ubicación de los senderos de muestreo en los bosques del PNNT y la RNNP, Guayana. PNNT = Parque Nacional Natural el Tuparro, RNNP = Reserva Nacional Natural Puinawai.

cálido, húmedo y ligeramente estacional, con un régimen de lluvias monomodal, con una precipitación promedio anual de 3,250 mm, un periodo máximo de lluvias entre mayo y agosto y uno mínimo entre diciembre y marzo. Las unidades de paisaje comprenden caatingas altas, bosques bajos sobre suelos mal drenados de las terrazas del río Inírida, bosques altos de superficie sedimentaria y áreas de colina. La zona se ubica dentro del ecosistema de zonobioma húmedo tropical del Guainía (Etter 2001; Romero et al. 2004).

La diversidad de plantas con flores en esta zona de estudio está concentrada en once familias (66.3\% de las especies), entre las que se encuentran Fabaceae (21 especies), Burseraceae (12), Moraceae (12), Lecythidaceae (11), Sapotaceae (11), Lauraceae (10). Las especies que se destacaron por su valor de importancia fueron Eperua leucantha, Eperua purpurea y Osteophloeum platyspermum, mientras que las familias con mayor importancia fueron Fabaceae, Sapotaceae y Lauraceae (Gómez 2010). El sitio de muestreo se ubicó en la zona aledaña a la comunidad indígena Zancudo, ubicada al norte de la RNNP. La comunidad de Zancudo está habitada por 
indígenas Puinaves, la cual está conformada por 160 habitantes aproximadamente agrupados en 40 familias (Tafur 2010). Las dos comunidades indígenas ubicadas en las áreas protegidas pueden realizan cacería de subsistencia porque pertenecen a los resguardo indígena dentro o en el área de influencia de las áreas protegidas (UAESPNN 1998)

Muestreo. Se obtuvo un mapa de coberturas por medio de una clasificación digital supervisada de las imágenes Landsat Path 4 Row 56 de enero 9 del 2001 para el sector nororiental del PNNT y Lansat Path 5 Row 58 del 14 de abril del 2001 para la RNNP. Para realizar los muestreos se seleccionaron los bosques de tierra firme para evitar la inundación durante la época de lluvias y que fueran accesibles a través de los ríos o caños. Todos los senderos de muestreo se hicieron para este estudio y ninguno de los lugares elegidos correspondió a caminos o senderos existentes previamente.

Cada sendero fue marcado y registrada la coordenada geográfica cada $50 \mathrm{~m}$. En el PNNT se abrieron seis senderos, tres en el sector del río Tomo y tres en el sector del río Tuparro, con una longitud de 2.15 a $4.5 \mathrm{~km}$ respectivamente y un total de $20 \mathrm{~km}$. En la RNNP se abrieron nueve senderos, tres en el sector de caño Zancudo y seis en ambos costados del río Inírida con una longitud de 3 a $5.5 \mathrm{~km}$ y un total de $33.3 \mathrm{~km}$ (Figura 1, Tabla 1). Todos los senderos se abrieron en conjunto con los pobladores de la comunidad Zancudo en la RNNP y con el personal técnico del PNNT. A lo largo de cada sendero se instalaron parcelas de huellas de $0.25 \mathrm{~m}^{2}$ cada 50 a $100 \mathrm{~m}$, para lo cual se eliminó toda la vegetación y se retiró el sustrato hasta una profundidad de $10 \mathrm{~cm}$, se eliminaron las raíces y se dejó el sustrato del suelo suelto para facilitar la impresión de las huellas. Después de cada revisión, cada parcela se preparó de nuevo borrando las huellas. Las características de los suelos fueron similares en todos los senderos. Se instalaron 211 parcelas de huellas en el PNNT y 297 en la RNNP.

Los muestreos se realizaron en paralelo en las dos áreas protegidas, con un equipo de tres investigadores por área, de tal manera que las observaciones se realizaron en simultáneo en tres senderos por cada área protegida durante 4 a 6 días consecutivos, hasta recorrer todos los senderos por área en cada mes de observaciones. Los muestreos se realizaron en dos meses durante el 2009 la primera sesión de muestreo fue del 24 abril al 20 mayo y la segunda durante julio, con una diferencia de 40 días entre las dos ocasiones de muestreo. Cada sendero se recorrió entre 9 y 12 días en total. Dado que las áreas de estudio se ubican en áreas bastante remotas y que la única forma de acceso es navegando por los ríos, el muestreo se realizó en la época de lluvias, durante la cual se facilita la navegación.

Tabla 1. Número de detecciones en el total de recorridos realizados en cada sendero. $\mathrm{AP}=$ Área protegida, $\mathrm{S}=\mathrm{Sendero}$, $\mathrm{Km}=$ Longitud del sendero, $\mathrm{Pa}=$ número de parcelas por sendero, $\mathrm{R}=$ total visitas a cada sendero, $\mathrm{EM}=$ kilómetros recorridos, $\mathrm{S}=$ Huellas en sendero, $\mathrm{P}=$ Huella en parcelas, $\mathrm{O}=$ Observación directa. $\mathrm{U}=$ conteo unificado considerando los tres métodos

\begin{tabular}{|c|c|c|c|c|c|c|c|c|c|c|c|c|c|c|c|c|c|c|c|c|c|c|c|c|c|c|}
\hline \multirow{2}{*}{ AP } & \multirow{2}{*}{$\mathbf{S}$} & \multirow{2}{*}{ Km } & \multirow{2}{*}{$\mathrm{Pa}$} & \multirow{2}{*}{$\mathbf{R}$} & \multirow{2}{*}{$\begin{array}{c}\text { EM } \\
(\mathrm{Km})\end{array}$} & \multicolumn{4}{|c|}{ Pecari tajacu } & \multicolumn{5}{|c|}{ T. pecari } & \multicolumn{4}{|c|}{ T. terrestris } & \multicolumn{4}{|c|}{ Mazama spp. } & \multicolumn{4}{|c|}{ O.virginianus } \\
\hline & & & & & & $\mathbf{S}$ & $\mathbf{P}$ & D & U & $\mathbf{S}$ & $\mathbf{P}$ & D & U & J & $\mathbf{S}$ & $\mathbf{P}$ & D & U & $\mathbf{S}$ & $\mathbf{P}$ & D & $\mathbf{U}$ & $\mathbf{S}$ & $\mathbf{P}$ & D & $\mathbf{U}$ \\
\hline \multirow{6}{*}{ PNNT } & A & 4.3 & 43 & 12 & 51.6 & 1 & 3 & & 4 & & & & & & & & & & & 3 & 2 & 5 & 1 & 1 & 2 & 4 \\
\hline & B & 2.2 & 21 & 12 & 25.8 & & 2 & 2 & 4 & & & & & & & & & & & 1 & & 1 & & & 1 & 1 \\
\hline & C & 3.7 & 38 & 12 & 44.4 & 4 & 3 & & 6 & 2 & 2 & & & 5 & 3 & & & 3 & 2 & 1 & & 3 & & & & \\
\hline & D & 3.0 & 42 & 11 & 33.0 & & & 1 & 1 & & & & & & 4 & 4 & & 8 & & 2 & & 2 & 2 & & 2 & 4 \\
\hline & $E$ & 4.5 & 45 & 11 & 49.5 & 2 & 3 & 1 & 5 & & & & & & 2 & & & 2 & 1 & 2 & 2 & 4 & 1 & & & 1 \\
\hline & $\mathrm{F}$ & 2.2 & 22 & 11 & 24.2 & 4 & 3 & 1 & 7 & & 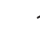 & & & 2 & 1 & & 1 & 2 & & 1 & & 1 & & & & \\
\hline Total & 6 & 20 & 211 & & 228.5 & 11 & 14 & 5 & 27 & 2 & 3 & & & 7 & 10 & 4 & 1 & 15 & 3 & 10 & 4 & 16 & & & & 10 \\
\hline
\end{tabular}


La búsqueda de ungulados se realizó mediante la observación directa de individuos y el registro indirecto a través de rastros. Los recorridos se realizaron mínimo 24 horas después de que los senderos fueron abiertos y las parcelas instaladas. Los observadores fueron rotados entre las dos áreas protegidas y entre los senderos para reducir los potenciales sesgos dependientes del observador (Peres 1999). Los recorridos de observación directa e indirecta se realizaron entre las 4:30 y las 14:30 h a una velocidad promedio de $1 \mathrm{~km} / \mathrm{h}$ con dos observadores por recorrido, un investigador y una persona local. El registro indirecto de ungulados se realizó mediante la búsqueda de rastros a lo largo de los senderos y en las parcelas de huellas. Cada huella encontrada en el sendero y/o en las parcelas se borró una vez anotada para evitar doble registro. El conjunto de huellas en forma de sendas se incluyó como uno sola y no se consideraron las huellas que no se pudieron identificar claramente para evitar falsas detecciones.

El conjunto de todas las detecciones obtenidas en cada recorrido se agruparon y se consideraron como un único registro por sendero, denotando 1 cuando se obtuvo alguna detección (huella y/o observación) y 0 en caso contrario. Se utilizó cada uno de los senderos como la unidad de muestreo. Para identificar las huellas se siguió la guía de Morales-Jiménez et al. (2004) y la experiencia de los guías locales.

Covariables. A partir de los mapas de coberturas obtenidos de la clasificación supervisada, se realizó un buffer de $1 \mathrm{~km}$ en cada sendero (1000 hectáreas en promedio) para obtener el porcentaje de cobertura de bosque alto y de bosque inundable en el paisaje circundante. Se calculó la distancia lineal hasta la comunidad indígena más cercana desde el centroide de cada sendero, la cual se denominó "distancia a poblados"y se utilizó como una medida indirecta de la presión de cacería. Los cálculos se realizaron en el software QGIS.

Análisis. Se realizó un análisis de varianza de una via para comparar el porcentaje de bosque total, bosque alto (BA) y bosque inundable (BI) entre las dos áreas protegidas, usando el área protegida como un factor. Se verificaron los supuestos de normalidad (prueba de Shapiro-wilk) y homogeneidad de varianzas (test de Levene). Se realizó una correlación de Spearman entre las covariables cuantitativas para analizar la multicolinearidad. En el análisis se consideraron las variables con un coeficiente de correlación $r<0.6$ y se incluyeron con una estandarización normalizada (dato original - media/ desviación estándar).

Se construyó un historial de detecciones con la información obtenida mediante la observación directa y el registro de huellas tomando cada sendero como un sitio para un total de 15 sitios. Se consideró cada día de recorrido como una visita por lo cual se obtuvieron entre 9 y 12 medidas repetidas, a partir de los cuales se generó un historial de detección para cada especie.

Se utilizó el modelo de ocupación con detección heterogénea desarrollado por Royle y Nichols (2003) (en adelante RN) quienes complementan el modelo original de MacKenzie et al. (2002) para estimar la abundancia a partir de datos de presencia y ausencia. En el modelo de RN la frecuencia de detección en cada sitio (i), es decir el número de veces que la especie fue detectada en los muestreos repetidos (j), sigue una distribución binomial con un número de intentos igual a (j) y una probabilidad (pi), la cual depende de una abundancia (Ni). La relación entre la probabilidad de detección por sitio (i) y la abundancia está dada por $\mathrm{p}_{\mathrm{ij}}=1-\left(1-\mathrm{r}_{\mathrm{ij}}\right)^{\mathrm{Ni}}$, en donde $\mathrm{p}_{\mathrm{ij}}$ es la probabilidad de detección de cada sitio, $r_{i j}$ es la probabilidad de detección propia de 
la especie y $\mathrm{N}_{\mathrm{i}}$ es el número de individuos en el sitio (Royle y Nichols 2003, Dénes et al. 2015). El modelo considera que la heterogeneidad en la probabilidad de detección específica de cada sitio está relacionada con la abundancia local. Esta relación entre la heterogeneidad en la probabilidad de detección causada por la variación en la abundancia local permite extraer información sobre la abundancia a partir de un estudio de ocupación mediante la mezcla de los modelos Binomial/Poisson (Dénes et al. 2015; Kery y Royle 2015).

Este modelo proporciona estimadores de los parámetros $\lambda$ y $r$, definidos como la abundancia promedio por sitio y la probabilidad de detección respectivamente (Royle y Nichols 2003). En el modelo de RN la probabilidad de ocupación ( $\Psi$ ) es un parámetro derivado que se estima como $\psi=1-\mathrm{e}^{-\lambda}$, en donde los sitios no ocupados tienen probabilidad de detección cero como resultado de abundancia cero. La probabilidad de ocupación se refiere a la proporción de sitios en los cuales la especie estuvo presente (Royle y Nichols 2003; Stanley y Royle 2005). Debido a que no podemos asegurar que las detecciones de las visitas repetidas corresponden a diferentes individuos de la población, los parámetros estimados de $\lambda$ no se interpretaron como abundancia absoluta por sitio sino como un índice de abundancia relativa ajustado por la detectabilidad (Stanley y Royle 2005; Thorn et al. 2011).

El modelo de RN asume que la población es cerrada, que los individuos se distribuyen al azar en el espacio y que la abundancia ( $\mathrm{Ni}$ ) se puede modelar con una distribución de probabilidad de Poisson (Royle y Nichols 2003). Este modelo ha sido usado para especies con bajas densidades como los ungulados y los carnívoros tropicales (Thorn et al. 2011; Gopalaswamy et al. 2012; Kalle et al. 2014). Se asumió que durante el periodo del estudio no hubo nacimientos ni muertes, por lo tanto, se consideraron como poblaciones cerradas durante el tiempo de muestreo.

El parámetro lambda $(\lambda)$ se expresó de forma relativa al esfuerzo de muestreo promedio por sendero $\left(\lambda / \mathrm{EM}_{\text {sendero }}\right)$, con lo cual se obtuvo un índice de abundancia relativa (Stanley y Royle 2005; Thorn et al. 2011; Gopalaswamy et al. 2012). La estimación de abundancia relativa $\lambda / \mathrm{EM}_{\text {sendero }}$ obtenida para los venados y el tapir se interpretó como el número de detecciones de individuos por kilómetro recorrido, mientras que para el pecarí de collar y el de labios blancos se interpretó como el número de detecciones de grupos por kilómetro recorrido.

La relación entre la abundancia relativa $(\lambda)$ y la probabilidad de detección ( $r$ ) con las covariables, se analizó mediante un modelo lineal generalizado con diferentes funciones de enlace (Dénes et al. 2015). La probabilidad de detección se analizó en función del esfuerzo de muestreo (EM) y del área protegida como un factor con dos niveles (AP). La abundancia relativa se analizó en función del área protegida como un factor (AP), el porcentaje de bosque alto (BA) y de bosque inundable en el paisaje (BI), y la distancia a poblados (DP). Para todos los casos, se realizó un modelo con cada covariable individual y el modelo saturado con todas las covariables.

El modelo detectabilidad para cada especie con todas las covariables analizadas fue: Logit $(r)=\beta_{0+} \beta_{1 *} A P+\beta_{2}{ }^{*}$ EM. El modelo de abundancia para cada especie con todas las covariables analizadas fue: $\log (\lambda)=\beta_{0+} \beta_{1^{*}} A P+\beta_{2}{ }^{*} B A+\beta_{3}{ }^{*} B I+\beta_{4}{ }^{*} D P$. El primer paso para construir los modelos candidatos a ser seleccionados fue analizar las covariables relacionadas con la probabilidad de detección, manteniendo constante la abundancia promedio por sitio $\lambda($.). Una vez encontrado el mejor modelo de detectabilidad, se construyeron los modelos de abundancia con el mejor 
modelo de detección. Se usaron los pesos de cada modelo derivados del criterio de información de Akaike corregidos para muestras pequeñas (AICC) para determinar la evidencia relativa a favor de cada modelo y determinar la influencia de cada una de las covariables (MacKenzie et al. 2006). Cuando varios modelos fueron igualmente posibles, se realizó un promedio de mismos para estimar el efecto (positivo o negativo) de las covariables a través de los coeficientes $(\beta)$. Se determinó que una covariable tuvo efecto en la abundancia y/o detectabilidad si los intervalos de confianza excluían el cero (90 \%) (Kery y Royle 2015). Se analizó el ajuste del modelo más parsimonioso para los datos observados a través de una función paramétrica de remuestreo que simula datos del modelo ajustado y aplica un ajuste de $x^{2}$ de Pearson (Fiske y Chandler 2011) y se estimaron los parámetros finales de abundancia ( $\lambda$ ) con los errores estándar asociados (Kalle et al. 2014). Todos los análisis estadísticos se realizaron en el programa estadístico R versión 3.2 (R Development Core Team 2012) con el paquete Unmarked (Fiske y Chandler 2011).

\section{Resultados}

En el PNNT se recorrieron $228.5 \mathrm{~km}$ (38 km en promedio por sendero) y en la RNNP $289.8 \mathrm{~km}$ ( $32 \mathrm{~km}$ en promedio por sendero). Mediante observación directa e indirecta en las dos áreas protegidas en el presente estudio se registraron cinco especies de ungulados: el tapir de tierras bajas, el pecarí de collar, el pecarí de labios blancos y los venados del género Mazama spp. (el venado pardo y el venado colorado). En el PNNT adicionalmente también se registró el venado cola blanca (Tabla 1).

Se obtuvieron 48 detecciones para el tapir, 15 en el PNNT y 33 en la RNNP. En cuanto a los tayassuidos, para el pecarí de collar se obtuvieron 52 detecciones, 27 en el PNNT y 25 en la RNNP, mientras que el pecarí de labios blancos se detectó en 13 ocasiones, 7 en el PNNT y 6 en la RNNP (Tabla 1). Durante las observaciones directas, el tamaño de los grupos de pecarí de collar fue de 10 en promedio y la única observación del pecarí de labios blancos fue de un grupo de al menos 20 individuos.

Las detecciones de los venados del género Mazama spp. correspondieron a las especies Mazama americana y Mazama nemorivaga, cuyas detecciones se agruparon a nivel de género, ya que no se logró discriminar entre las especies a través de los registros obtenidos con las huellas. Se obtuvieron 31 registros de Mazama spp., 16 en el PNNT y 15 en la RNNP. El venado cola blanca se detectó únicamente en el PNNT con 10 registros (Tabla 1).

Covariables. No se encontró correlación entre el porcentaje de bosque alto y el porcentaje de bosque inundable en el paisaje circundante a cada sendero $(r=-0.3)$, ni entre la distancia a poblados y el porcentaje de bosque alto $(r=-0.5)$ y bosque inundable $(r=-0.26)$. Por lo tanto, las tres covariables se incluyeron en el análisis. El porcentaje promedio de bosque en el paisaje circundante a cada sendero (buffer de 1000 ha) mostró diferencia significativa entre las áreas $(F=139.4, \mathrm{~g} . \mathrm{l} .=13, P<0.05)$ y fue el doble para la RNNP (89.2\%) respecto al PNNT (45.3\%). Esta misma relación se mantuvo analizando separadamente el bosque alto (RNNP $=75.2 \%$, PNNT $=36.3$ $\% ; F=1.01, \mathrm{~g} . \mathrm{l} .=13, P=0.33$ ) y el bosque inundable (RNNP $=14.1 \%, \mathrm{PNNT}=8.9 \%$; $\mathrm{F}=43.3$, g. I. $=13, P>0.05$ ), aunque este último no presentó diferencia significativa.

Abundancia: modelo de ocupación con heterogeneidad en la detección. Se encontró buen ajuste del modelo más parsimonioso para todas las especies (tapir $P=0.6$, pecarí de collar $P=0.5$, pecarí de labios blancos $P=0.7$, venados Mazama spp. $P=0.6$, venado 
cola blanca $P=0.6$ ). El peso de los modelos candidatos (w) fue homogéneo en la mayoría de los casos indicando que varios de los modelos analizados fueron igualmente posibles (Tabla 2), por lo tanto, se realizó un promedio de los mismos (Tabla 3).

Se encontró que las abundancias relativas del tapir, el pecarí de collar y de los venados del género Mazama spp. fueron diferentes entre las áreas protegidas mientras que no se observó diferencia en la abundancia del pecarí de labios blancos (Tabla 3). La estimación de abundancia relativa ajustada por el esfuerzo de muestreo promedio por sendero $\left(\lambda / \mathrm{EM}_{\text {promedio }}\right.$ ) fue mayor en la RNNP para el tapir (RNNP $=0.08$, PNNT $=0.06)$ y Mazama spp. $($ RNNP $=0.43$, PNNT $=0.27)$ contrario a lo encontrado para el pecarí de collar (RNNP $=0.28$, PNNT $=0.42$ ). La abundancia relativa del pecarí de labios blancos fue de 0.02 para las dos áreas. La abundancia relativa del venado cola blanca fue 0.1 para el PNNT.

El porcentaje de bosque alto no tuvo efecto en la abundancia de ninguna especie de ungulado. El porcentaje de bosque inundable en el paisaje tuvo efecto positivo

Tabla 2. Modelos de ocupación con heterogeneidad en la detección para ungulados en dos áreas protegidas de la Guyana colombiana a partir del registro de huellas y observaciones directas. Los parámetros $\lambda$ y $r$ son definidos como la abundancia relativa promedio por sitio y la probabilidad de detección respectivamente (Royle y Nichols 2003). Los modelos incluyen el área protegida como factor (AP), el esfuerzo de muestreo (EM), el porcentaje de cobertura de bosque alto (BA), el porcentaje de bosque inundable (BI) y la distancia a poblados (DP). N.P.: Número de parámetros, AICc: Criterio de Información de Akaike, $\triangle$ QAICc: diferencia en los valores de AICc entre los modelos, w: peso del modelo.

\begin{tabular}{|c|c|c|c|c|}
\hline Modelo & N.P. & AICc & $\Delta \mathrm{AICC}$ & $\mathbf{w}$ \\
\hline \multicolumn{5}{|l|}{ Tapirus terrestris } \\
\hline$\lambda(\mathrm{DP}), r(\mathrm{EM})$ & 4 & 180.0 & 0.55 & 0.6 \\
\hline$\lambda(),. r()$. & 2 & 182.8 & 0.13 & 0.7 \\
\hline$\lambda(\mathrm{BI}), r(\mathrm{EM})$ & 4 & 183.6 & 0.09 & 0.8 \\
\hline$\lambda(\mathrm{AP}), \mathrm{r}(\mathrm{EM})$ & 4 & 183.6 & 0.09 & 0.9 \\
\hline$\lambda(\mathrm{BA}), \mathrm{r}(\mathrm{EM})$ & 4 & 183.9 & 0.08 & 0.9 \\
\hline$\lambda(A P+B A+B I+D P), r(E M)$ & 7 & 184.3 & 0.06 & 1.0 \\
\hline \multicolumn{5}{|l|}{ Pecari tajacu } \\
\hline$\lambda(\mathrm{DP}), \mathrm{r}()$. & 3 & 201.4 & 0.32 & 0.3 \\
\hline$\lambda(),. r()$. & 2 & 201.9 & 0.25 & 0.6 \\
\hline$\lambda(\mathrm{AP}), r()$. & 3 & 202.7 & 0.17 & 0.7 \\
\hline$\lambda(B A), r()$. & 3 & 203.1 & 0.14 & 0.9 \\
\hline$\lambda(\mathrm{BI}), \mathrm{r}()$. & 3 & 203.9 & 0.09 & 1.0 \\
\hline$\lambda(\mathrm{AP}+\mathrm{BA}+\mathrm{BI}+\mathrm{DP}), \mathrm{r}()$. & 6 & 206.1 & 0.03 & 1.0 \\
\hline \multicolumn{5}{|l|}{ Tayassu pecari } \\
\hline$\lambda(\mathrm{BI}), r(\mathrm{EM})$ & 4 & 84.9 & 0.43 & 0.4 \\
\hline$\lambda(\mathrm{AP}), \mathrm{r}(\mathrm{EM})$ & 4 & 86.2 & 0.23 & 0.7 \\
\hline$\lambda(),. r()$. & 2 & 87.7 & 0.11 & 0.8 \\
\hline$\lambda(\mathrm{DP}), \mathrm{r}(\mathrm{EM})$ & 4 & 87.9 & 0.10 & 0.9 \\
\hline$\lambda(B A), r(E M)$ & 4 & 88.1 & 0.09 & 1.0 \\
\hline$\lambda(\mathrm{AP}+\mathrm{BA}+\mathrm{BI}+\mathrm{DP}), r(\mathrm{EM})$ & 7 & 89.1 & 0.05 & 1.0 \\
\hline \multicolumn{5}{|l|}{ Mazama spp. } \\
\hline$\lambda(D P), r(E M)$ & 4 & 156.6 & 0.34 & 0.3 \\
\hline$\lambda(\mathrm{AP}), r(\mathrm{EM})$ & 4 & 157.8 & 0.19 & 0.5 \\
\hline$\lambda(\mathrm{BI}), \mathrm{r}(\mathrm{EM})$ & 4 & 158.0 & 0.17 & 0.7 \\
\hline$\lambda(B A), r(E M)$ & 4 & 158.3 & 0.15 & 0.9 \\
\hline$\lambda(),. r()$. & 2 & 158.6 & 0.13 & 1.0 \\
\hline$\lambda(\mathrm{AP}+\mathrm{BA}+\mathrm{BI}+\mathrm{DP}), r(\mathrm{EM})$ & 7 & 162.3 & 0.02 & 1.0 \\
\hline \multicolumn{5}{|l|}{ Odocoileus virginianus } \\
\hline$\lambda(\mathrm{DP}), \mathrm{r}()$. & 4 & 85.8 & 0.52 & 0.5 \\
\hline$\lambda(),. r()$. & 2 & 88.0 & 0.17 & 0.7 \\
\hline$\lambda(B \mathrm{I}), \mathrm{r}()$. & 4 & 88.8 & 0.11 & 0.8 \\
\hline$\lambda(B A+B I+D P), r()$. & 4 & 89.1 & 0.10 & 0.9 \\
\hline$\lambda(B A), r()$. & 6 & 89.2 & 0.10 & 1.0 \\
\hline
\end{tabular}


en la abundancia del pecarí de labios blancos. La distancia a poblados tuvo un efecto positivo en la abundancia del pecarí de collar (mayor abundancia cuando la distancia es mayor), mientras que el efecto fue contrario para el tapir y el venado cola blanca (Tabla 3).

La ocupación del tapir fue de $\Psi=0.56$ en el PNNT y de $\Psi=0.68$ en la RNNP y para el pecarí de collar fue $\Psi=0.93$ en el PNNT y $\Psi=0.89$ en la RNNP (Tabla 3). El pecarí de labios blancos tuvo una ocupación de $\Psi=0.51$ para el PNNT y $\Psi=0.44$ para RNNP. Por

Tabla 3. Coeficientes del modelo promedio para cinco especies de ungulados basados en el registro de huellas y observación directa en la Guayana colombiana. Los parámetros $\lambda$, ry $(\psi)$, son definidos como la abundancia relativa promedio, la probabilidad de detección y la probabilidad de ocupación (Royle y Nichols 2003). Beta $(\beta)=$ coeficiente, $E E=$ error estándar, $5 \%$ = límite inferior de intervalo de confianza, $90 \%$ = límite superior de intervalo de confianza.

\begin{tabular}{|c|c|c|c|c|c|c|c|c|}
\hline \multicolumn{2}{|c|}{$\begin{array}{l}\text { Especie - } \\
\text { Parámetro }\end{array}$} & Beta $(\beta)$ & EE & $5 \%$ & $90 \%$ & $\lambda(\mathrm{EE})$ & $r(E E)$ & $\psi$ \\
\hline \multirow{8}{*}{ 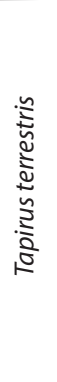 } & $\lambda$ (PNNT) & 0.83 & 0.80 & -0.49 & 2.15 & $2.3(1.8)$ & $0.1(0.07)$ & 0.56 \\
\hline & $\lambda(\mathrm{RNNP})$ & 1.14 & 0.54 & 0.25 & 2.03 & 3.1 (1.69) & & 0.68 \\
\hline & $\lambda(\mathrm{BA})$ & 0.05 & 0.26 & -0.38 & 0.47 & & & \\
\hline & $\lambda(\mathrm{BI})$ & 0.11 & 0.21 & -0.23 & 0.45 & & & \\
\hline & $\lambda(\mathrm{DP})$ & -0.63 & 0.37 & -1.25 & -0.02 & & & \\
\hline & $\lambda(\operatorname{lnt})$ & 0.94 & 0.58 & -0.02 & 1.89 & & & \\
\hline & $\mathrm{p}(\mathrm{EM})$ & -0.22 & 0.41 & -0.90 & 0.45 & & & \\
\hline & $\mathrm{p}$ (Int) & -1.78 & 0.67 & -2.88 & -0.69 & & & \\
\hline \multirow{7}{*}{ 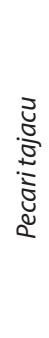 } & $\lambda($ PNNT) & 2.65 & 0.56 & 1.73 & 3.57 & $14.6(6.8)$ & $0.09(0.05)$ & 0.93 \\
\hline & $\lambda($ RNNP) & 2.32 & 0.61 & 1.31 & 3.32 & $10.4(5.5)$ & $0.09(0.05)$ & 0.89 \\
\hline & $\lambda(\mathrm{BA})$ & -0.13 & 0.17 & -0.41 & 0.15 & & & \\
\hline & $\lambda(\mathrm{BI})$ & 0.01 & 0.17 & -0.26 & 0.29 & & & \\
\hline & $\lambda(\mathrm{DP})$ & 0.23 & 0.15 & 0.01 & 0.47 & & & \\
\hline & $\lambda($ Int $)$ & 2.46 & 0.73 & 1.26 & 3.65 & & & \\
\hline & $\mathrm{p}(\operatorname{Int})$ & -3.32 & 0.73 & -4.53 & -2.12 & & & \\
\hline \multirow{8}{*}{ 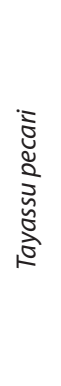 } & $\lambda($ PNNT) & -0.71 & 0.75 & -1.95 & 0.53 & $0.8(0.5)$ & $0.04(0.02)$ & 0.51 \\
\hline & $\lambda(\mathrm{RNNP})$ & 0.59 & 0.72 & -0.59 & 1.77 & $0.8(0.5)$ & $0.04(0.02)$ & 0.44 \\
\hline & $\lambda(\mathrm{BA})$ & 0.20 & 0.47 & -0.58 & 0.98 & & & \\
\hline & $\lambda(\mathrm{BI})$ & 0.70 & 0.38 & 0.07 & 1.32 & & & \\
\hline & $\lambda(\mathrm{DP})$ & -0.30 & 0.47 & -1.07 & 0.47 & & & \\
\hline & $\lambda(\operatorname{lnt})$ & -0.08 & 0.59 & -1.05 & 0.89 & & & \\
\hline & $\mathrm{p}(\mathrm{EM})$ & 1.22 & 0.54 & 0.34 & 2.10 & & & \\
\hline & $\mathrm{p}$ (Int) & -2.28 & 0.63 & -3.31 & -1.25 & & & \\
\hline \multirow{8}{*}{ 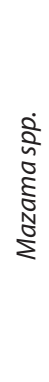 } & $\lambda(\mathrm{PNNT})$ & 2.26 & 0.63 & 1.23 & 3.29 & $9.5(5.9)$ & $0.02(0.01)$ & 0.89 \\
\hline & $\lambda$ (RNNP) & 2.70 & 0.37 & 2.09 & 3.30 & $14.9(5.5)$ & $0.02(0.01)$ & 0.93 \\
\hline & $\lambda(\mathrm{BA})$ & 0.09 & 0.23 & -0.29 & 0.48 & & & \\
\hline & $\lambda(\mathrm{BI})$ & 0.13 & 0.20 & -0.21 & 0.46 & & & \\
\hline & $\lambda(\mathrm{DP})$ & -0.32 & 0.25 & -0.73 & 0.10 & & & \\
\hline & $\lambda($ Int $)$ & 2.49 & 0.85 & 1.09 & 3.90 & & & \\
\hline & $p(E M)$ & 0.49 & 0.24 & 0.10 & 0.88 & & & \\
\hline & $\mathrm{p}(\operatorname{lnt})$ & -4.03 & 0.84 & -5.41 & -2.65 & & & \\
\hline \multirow{5}{*}{$\begin{array}{l}\frac{n}{2} \\
\stackrel{5}{5} \\
\stackrel{5}{5} \\
.5 \\
\vdots \\
0\end{array}$} & $\lambda(\mathrm{BA})$ & 0.04 & 0.42 & -0.66 & 0.73 & & & \\
\hline & $\lambda(\mathrm{BI})$ & -0.22 & 0.48 & -1.01 & 0.57 & & & \\
\hline & $\lambda(\mathrm{DP})$ & -0.80 & 0.42 & -1.50 & -0.11 & & & \\
\hline & $\lambda(\operatorname{lnt})$ & 0.57 & 0.88 & -0.88 & 2.01 & $3.48(6.3)$ & $0.03(0.06)$ & 0.43 \\
\hline & $\mathrm{p}$ (Int) & -2.38 & 0.94 & -3.94 & -0.83 & & & \\
\hline
\end{tabular}


último, para Mazama spp. se obtuvo una ocupación $\Psi=0.89$ en el PNNT y $\Psi=0.93$ en la RNNP. La ocupación del venado cola blanca en el PNNT fue $\Psi=0.43$.

Se observó diferencia en la probabilidad de detección entre las especies de ungulados analizadas. El esfuerzo de muestreo (EM) tuvo efecto en la detectabilidad para el pecarí de labios blancos y Mazama spp. (Tabla 3). La probabilidad de detección fue muy baja para todas las especies, siendo Mazama spp. el que presentó la menor probabilidad de detección, seguido por el venado cola blanca, el pecarí de labios blancos, el pecarí de collar y por último el tapir.

\section{Discusión}

Se encontró diferencia en la abundancia de ungulados entre las dos áreas protegidas para tres especies de ungulados. Los modelos de RN permitieron estimar la abundancia relativa para las cinco especies de ungulados aun con la baja detectabilidad observada la cual está asociada con sus bajas abundancias (Tabla 3). Simulaciones realizadas para los modelos de ocupación con heterogeneidad en la detección indican que para las situaciones con pocos sitios (menores a 25) y con baja probabilidad de detección ( $r<=0.1$ ), los sesgos pueden ser del orden de 10 a $15 \%$ (Royle y Nichols 2003; Stanley y Royle 2005; Thorn et al. 2011). Para estos casos, recomiendan un incremento en el esfuerzo de muestreo en las medidas repetidas de 9 a 12 por sitio (Thorn et al. 2011). En nuestro caso, las medidas repetidas fueron entre 9 y 12 por sendero, con por lo cual sugerimos que las estimaciones son confiables a pesar de los pocos sitios (15) y la baja detectabilidad de las especies (Tabla 3), por lo cual el sesgo en nuestras estimaciones podría ser del 10 a $15 \%$.

El tapir es una especie dependiente del bosque (Desbiez et al. 2009) de tal manera que su presencia ha estado asociada positivamente con la cantidad de bosque disponible en el paisaje (Tejeda-Cruz et al. 2009; Garmendia et al. 2013) y la probabilidad de ocurrencia y la abundancia aumentan a medida que la cantidad de bosque continuo aumenta. Esta misma relación en la preferencia por paisajes con mayor cobertura de bosque ocurre para los venados Mazama spp. (Sampaio et al. 2010). El venado pardo es más común y ocupa ampliamente todos los tipos de bosque, los arbustales y los ambientes abiertos (Ferreguetti et al. 2015), mientras que el colorado muestra preferencia por el bosque maduro y continuo (Rivero et al. 2005; Desbiez et al. 2009). Si bien, la abundancia para ambas especies, el tapir y los venados Mazama spp., fue mayor en la RNNP, no encontramos relación entre la cantidad de bosque en el paisaje y la abundancia. Por lo tanto, la mayor abundancia en la RNNP puede estar relacionada con las condiciones del hábitat a escala local tales como la disponibilidad de salados o de alimento (Bodmer 1989; Tobler et al. 2009; Tobler et al. 2010). El venado cola blanca es una especie que prefiere áreas abiertas y evita el bosque maduro el cual usa como refugio (Tejeda-Cruz et al. 2009), lo cual se ve reflejado en la ocupación del $43 \%$ de los sitios con bosque en el PNNT.

El pecarí de labios blancos es otra especie sensible a la cantidad de bosque en el paisaje. Esta especie se ha encontrado con preferencia en bosque continuo y en parches de bosque de gran tamaño (Michalski y Peres 2007; Garmendia et al. 2013). Es una especie demandante de grandes áreas dado que vive en grupos numerosos que requieren áreas de acción de gran magnitud (Fragoso 1998; Keuroghlian et al. 2004; Reyna-Hurtado et al. 2009). También se ha encontrado que los bosques ribereños funcionan como corredores naturales ya que los pecaríes de labios blancos recorren 
grandes distancias a través del paisaje para obtener los recursos necesarios para mantener sus grandes tropas (Fragoso 1999) o utilizan estacionalmente los recursos alimenticios disponibles en estos bosques ribereños (Keuroghlian et al. 2009). Por lo general, estos pecaríes no se registran en corredores de bosque aislados (Lees y Peres 2008). Por lo tanto, el no encontrar diferencia en la abundancia de esta especie entre las dos áreas protegidas, puede ser un indicador de la buena condición de los bosques en las dos áreas protegidas, los cuales proporcionan el hábitat adecuado para esta especie, a pesar de las diferencias en la proporción de bosque en el paisaje. Por esta razón, sugerimos que la presencia de estos pecaríes en las dos áreas estudiadas es un buen indicador del buen estado de conservación de sus bosques pues es una especie selectiva de bosques prístinos (Desbiez et al. 2009).

La relación positiva de la abundancia del pecarí de labios blancos y el porcentaje de bosque inundable en el paisaje, coincide con los patrones observados en otras áreas de la Amazonía (Bodmer 1990; Tobler et al. 2009). Los pecaríes de labios blancos migran entre el bosque de tierra firme y el bosque inundable y les permite aprovechar la mayor producción de frutos del bosque inundable (Bodmer 1990).

De otra parte, la abundancia del pecarí de collar se ha encontrado asociada de manera positiva y beneficiada por un grado moderado de subdivisión (número de parches) del bosque en el paisaje (Michalski y Peres 2007; Tejeda-Cruz et al. 2009; Thornton et al. 2011; Garmendia et al. 2013). Esto podría explicar la mayor abundancia del pecarí de collar en el PNNT. En el PNNT el bosque se encuentra disponible de manera natural en parches y esta configuración parece favorecer a esta especie, puesto que también puede usar los recursos de áreas abiertas (Keuroghlian y Eaton 2008; Tejeda-Cruz et al. 2009). Sin embargo, no se observó efecto entre la cantidad de bosque en el paisaje y la abundancia para esta especie.

El uso de hábitat del tapir incrementa con el incremento en la protección contra cacería y el incremento en la distancia a puntos de acceso para cazadores (Cruz et al. 2014), por lo tanto, la relación negativa de la distancia a poblados $(\beta=-0.63)$ puede reflejar la baja presión de cacería para esta especie. Esto coincide con que el tapir no se reportó como una de las especies de cacería de subsitencia en las comunidades del PNNT (Martinez-Salas et al. 2016) y en la RNNP el aprovechamiento fue muy bajo con una frecuencia de 1.4 individuos por año (Tafur 2010). Esta misma tendencia se observó para el venado cola blanca en el PNNT, indicando baja presión de cacería en el PNNT. Contrario a esto, el efecto positivo de la distancia a poblados para el pecarí de collar, puede estar relacionado con la presión de cacería de subsistencia de las comunidades locales, en donde el pecarí de collar representó el mayor aporte de biomasa a las comunidades del Resguardo de Curripacos (Martinez-Salas et al. 2016).

Las limitaciones del trabajo están relacionadas a que nuestras estimaciones de abundancia relativa corresponden a una sola época climática. Así mismo, los datos obtenidos corresponden a esta zona estudiada y sería difícil extrapolar a áreas con condiciones de hábitat diferente. Las limitaciones metodológicas de este trabajo están relacionadas con la baja detectabilidad de los métodos usados en la época de Iluvia, lo cual tratamos de resolver con modelos que tienen en cuenta de detección imperfecta. Es posible que la detección mejore con métodos como cámaras trampa.

De acuerdo a nuestros resultados, estas áreas protegidas estudiadas son muy relevantes porque albergan poblaciones vulnerables de estos ungulados, ya que el PNNT es la única área protegida del sistema nacional que incluye ecosistemas de 
pastizal y la RNNP se encuentra altamente vulnerable por la actividad de minería ilegal (Güiza 2014). Los modelos de ocupación permitieron discriminar diferencias entre zonas, aun en condiciones de bajas abundancias y pueden convertirse en una herramienta fundamental para el monitoreo de estas poblaciones a largo plazo. Tales monitoreos son necesarios para evaluar la efectividad de las áreas protegidas en la conservación de los ungulados, especialmente en la región del escudo guayanés, únicas por su antigüedad y singularidad.

\section{Agradecimientos}

Agradecemos al Grupo Conservación y Manejo de Vida Silvestre del Instituto de Ciencias de la Universidad Nacional de Colombia (UN), a la Unidad Administrativa Especial del Sistema de Parques Nacionales Naturales, específicamente el PNNT y la RNNP. Este trabajo fue financiado por Colciencias a través del Proyecto "Ecología, uso y conservación de ungulados en la Orinoquía y Amazonía colombianas", la Fundación Alejandro Ángel Escobar - Beca Colombia Biodiversa y la División de Investigación-Sede Bogotá-UN. Agradecemos a nuestros colegas M. Rodríguez, N. Roncancio, M. Martínez, F. Suárez, W. López y C. Mora por el trabajo de campo. También agradecemos a todos los miembros de la comunidad Zancudo y al personal del PNNT por la colaboración en campo. A J. Thompson por su valiosa colaboración con los análisis de ocupación. Finalmente, agradecemos a los evaluadores y al editor asociado por los valiosos aportes que enriquecieron el manuscrito.

\section{Literatura citada}

Altrichter, M., y G. I. Boaglio. 2004. Distribution and relative abundance of peccaries in the Argentine Chaco: associations with human factors. Biological Conservation 116:217-225.

Altrichter, M., A. Taber, H. Beck, R. Reyna-Hurtado, L. Lizarraga, A. Keuroghlian, y E.W. Sanderson. 2012. Range-wide declines of a key Neotropical ecosystem architect, the Near Threatened white-lipped peccary Tayassu pecari. ORYX 46:87-98.

Beck, H., J. W. Snodgrass, y P. Thebpanya. 2013. Long-term exclosure of large terrestrial vertebrates: Implications of defaunation for seedling demographics in the Amazon rainforest. Biological Conservation 163:115-121.

Bodmer, R. E. 1989. Frugivory in Amazonian Artiodactyla: evidence for the evolution of the ruminant stomach. Journal of Zoology 219:457-467.

Bodmer, R. E. 1990. Responses of ungulates to seasonal inundations in the Amazon Floodplain. Journal of Tropical Ecology 6:191-201.

Burnham, K. P., D. R. Anderson, y J. L. Laake. 1980. Estimation of density from line transect sampling of biological populations. Wildlife Monographs 72:3-202.

Correa, H. D., S. L. Ruiz, y L. M. Arévalo (eds.). 2006. Plan de acción en biodiversidad de la cuenca del Orinoco-Colombia/2005-2015: Propuesta técnica. Corporinoquia, Cormacarena, IAvH, Unitrópico, Fundación Omacha, Fundación Horizonte Verde, Universidad Javeriana, Unillanos, WWF-Colombia, GTZ-Colombia. Bogotá, Colombia.

Cruz, P., A. Paviolo, R. F. Bó, J. J. Thompson, y M. S. Di Bitetrı. 2014. Daily activity patterns and habitat use of the lowland tapir (Tapirus terrestris) in the Atlantic Forest. Mammalian Biology 79:376-383.

De Thoisy, B., S. Brosse, y M. A. Dubois. 2008. Assessment of large-vertebrate species richness and relative abundance in Neotropical forest using line-transect censuses: what is the minimal effort required? Biodiversity and Conservation 17:2627-2644.

de Thoisy, B., C. Richard-Hansen, B. Goguillon, P. Joubert, J. Obstancias, P. Winterton, y S. Brosse. 2010. Rapid evaluation of threats to biodiversity: human footprint score and large vertebrate species responses in French Guiana. Biodiversity and Conservation 19:1567-1584. 
Dénes, F. V., L. F. SiLveiRA, y S. R. Beissinger. 2015. Estimating abundance of unmarked animal populations: accounting for imperfect detection and other sources of zero inflation. Methods in Ecology and Evolution 6:543-556.

Desbiez, A. L. J., R. E. Bodmer, y S. A. Santos. 2009. Wildlife habitat selection and sustainable resources management in a Neotropical wetland. International Journal of Biodiversity and Conservation 1:11-20.

Dirzo, R., H. S. Young, M. Galetti, G. Ceballos, N. J. Isaac, y B. Collen. 2014. Defaunation in the Anthropocene. Science 345:401-406.

Duarte, J. M., y A. Voglotti. 2015. Mazama americana. The IUCN Red List of Threatened Species. Disponible en: http://www.iucnredlist.org/details/29619/0.

Etter, A. 2001. Puinawai y Nukak: Caracterización ecológica de dos reservas nacionales naturales de la Amazonía colombiana. Pontificia Universidad Javeriana. Bogotá, Colombia.

Ferreguetti, Á. C., W. M. Tomás, y H. G. Bergallo. 2015. Density, occupancy, and activity pattern of two sympatric deer (Mazama) in the Atlantic Forest, Brazil. Journal of Mammalogy 96:1245-1254.

FisKe, I., Y R. ChANDler. 2011. Unmarked: An R package for fitting hierarchical models of wildlife occurrence and abundance. Journal of Statistical Software 43:1-23.

ForGet, P. M., Y D. S. Hammond. 2005. Rainforest vertebrates and food plant diversity in the Guiana Shield. Pp. 233-294 in Tropical forests of the Guiana shield: ancient forests in a modern world. CABI International. Wallington, Reino Unido.

Fragoso, J. M. V. 1998. Home Range and Movement Patterns of White-lipped Peccary (Tayassu pecari) herds in the Northern Brazilian Amazon. Biotropica 30:458-469.

Fragoso, J. M. V. 1999. Perception of scale and resource partitioning by peccaries: behavioral causes and ecological implications. Journal of Mammalogy 80:993-1003.

Gabor, T. M., y E. C. Hellgren. 2000. Variation in peccary populations: landscape composition or competition by an invader? Ecology 81:2509-2524.

Gabor, T. M., E. C. Hellgren, y N. J. Silvy. 2001. Multi-scale habitat partitioning in sympatric suiforms. The Journal of Wildlife Management 65:99-110.

Garmendia, A., V. Arroyo-Rodríguez, A. Estrada, E. J. Naranjo, y K. E. Stoner. 2013. Landscape and patch attributes impacting medium and large-sized terrestrial mammals in a fragmented rain forest. Journal of Tropical Ecology 29:331-344.

Gómez, B. 2010. Densidad y áreas de ocupación del pecarí de collar (Pecari tajacu) y su relación con el hábitat en el Parque Nacional Natural Tuparro y la Reserva Natural Puinawai. Tesis de maestria en Ciencias Biología. Facultad de Ciencias, Universidad Nacional de Colombia. Bogotá, Colombia.

Gómez, B., y O. Montenegro. 2012. Abundancia del pecarí de collar (Pecari tajacu) en dos áreas protegidas de la Guayana colombiana. Mastozoología Neotropical 19:163-178.

Gopalaswamy, A. M., K. U. Karanth, N. S. Kumar, y D. W. Macdonald. 2012. Estimating tropical forest ungulate densities from sign surveys using abundance models of occupancy. Animal Conservation 15:669-679.

Güıza, L. 2014. La realidad de la minería ilegal en países amazónicos. Sociedad Peruana de Derecho Ambiental. Lima, Perú.

Hammond, D. S. 2005. Ancient land in a modern world. Pp. 1-14 in Tropical forests of the Guiana shield: ancient forests in a modern world. CABI International. Wallington, UK.

Harrison, R. D., S. Tan, J. B. Plotkin, F. Slik, M. Detto, T. Brenes, A. Itoh, y S. J. Davies. 2013. Consequences of defaunation for a tropical tree community. Ecology Letters 16:687-694.

Kalle, R., T. Ramesh, Q. Qureshi, y K. Sankar. 2014. Estimating seasonal abundance and habitat use of small carnivores in the Western Ghats using an occupancy approach. Journal of Tropical Ecology 30:469-480.

KerY, M., y J. A. Royle. 2015. Applied Hierarchical Modeling in Ecology: Analysis of distribution, abundance and species richness in R and BUGS: Volume 1: Prelude and Static Models. Academic Press. Londres, Reino Unido. 
Keuroghlian, A., y D. P. Eaton. 2008. Importance of rare habitats and riparian zones in a tropical forest fragment: preferential use by Tayassu pecari, a wide-ranging frugivore. Journal of Zoology 275:283-293.

Keuroghlian, A., D. P. Eaton, y A. L. Desbiez. 2009. The response of a landscape species, white-lipped peccaries, to seasonal resource fluctuations in a tropical wetland, the Brazilian Pantanal. International Journal of Biodiversity and Conservation 1:87-97.

Keuroghlian, A., D. P. Eaton, y W. S. Longland. 2004. Area use by white-lipped and collared peccaries (Tayassu pecari and Tayassu tajacu) in a tropical forest fragment. Biological Conservation 120:411-425.

Keuroghlian, A., R. Reyna-Hurtado, M. Altrichter, H. Beck, A. Taber, y J. M. V. Fragoso. 2013. Tayassu pecari. The IUCN Red List of Threatened Species. Disponible en: http://www.iucnredlist.org/ details/41778/0.

KuRTen, E.L. 2013. Cascading effects of contemporaneous defaunation on tropical forest communities. Biological Conservation 163:22-32.

Lees, A. C., y C. A. Peres. 2008. Conservation value of remnant riparian forest corridors of varying quality for Amazonian birds and mammals. Conservation Biology 22:439-449.

MacKenzie, D. I., y J. D. Nichols. 2004. Occupancy as a surrogate for abundance estimation. Animal Biodiversity and Conservation 27:461-467.

MacKenzie, D. I., J. D. Nichols, G. B. Lachman, S. Droege, J. Andrew Royle, y C. A. Langtimm. 2002. Estimating site occupancy rates when detection probabilities are less than one. Ecology 83:2248-2255.

MacKenzie, D. I., J. D. Nichols, J. A. Royle, K. H. Pollock, L. L. Bailey, y J. E. Hines. 2006. Occupancy estimation and modeling. Academic Press. California, EE. UU.

Martínez-Salas, M., H. F. López-Arévalo, y P. Sánchez-Palomino. 2016. Cacería de subsistencia de mamíferos en el sector oriental de la Reserva de Biósfera El Tuparro, Vichada (Colombia). Acta Biológica Colombiana 21:151-166.

Michalski, F., y C. A. Peres. 2007. Disturbance-mediated mammal persistence and abundance-area relationships in Amazonian forest fragments. Conservation Biology 21:1626-1640.

Morales-Jiménez, A. L., K. Poveda, y F. Sánchez. 2004. Mamíferos terrestres y voladores de Colombia: Guía de campo. Bogotá, Colombia.

Morellet, N., J. M. Gaillard, A. J. M. Hewison, P. Ballon, Y. Boscardin, P. Duncan, F. Klein, y D. Maillard. 2007. Indicators of ecological change: new tools for managing populations of large herbivores. Journal of Applied Ecology 44:634-643.

Munari, D. P., C. Keller, y E. M. Venticinque. 2011. An evaluation of field techniques for monitoring terrestrial mammal populations in Amazonia. Mammalian Biology 76:401-408.

Naranjo, E. J., y R. E. Bodmer. 2007. Source-sink systems and conservation of hunted ungulates in the Lacandon Forest, Mexico. Biological Conservation 138:412-420.

Naveda, A., B. de Thoisy, C. Richard-Hansen, D. A. Torres, L. Salas, R. Wallance, S. Chalukian, y S. de Bustos. 2008. Tapirus terrestris. The IUCN Red List of Threatened Species. Disponible en: http://www. iucnredlist.org/details/21474/0.

Patterson, B. D., y L. P. Costa. 2012. Bones, Clones, and Biomes: The History and Geography of Recent Neotropical Mammals. University of Chicago Press. Chicago, EE. UU.

Peres, C. A. 1996. Population status of white-lipped Tayassu pecari and collared peccaries T. tajacu in hunted and unhunted Amazonian forests. Biological Conservation 77:115-123.

Peres, C. A. 1999. General guidelines for standardizing line-transect surveys of tropical forest primates. Neotropical Primates 7:11-16.

Peres, C.A., y E. Palacios. 2007. Basin-wide wffects of game harvest on vertebrate population densities in Amazonian Forests: Implications for animal-mediated seed sispersal. Biotropica 39:304-315.

R Development Core Team. 2012. R: A language and environment for statistical computing. R Foundation for Statistical Computing. Vienna, Austria. 
Ray, J., K.H. Redford, R. Steneck, y J. Berger. 2013. Large carnivores and the conservation of biodiversity. Island Press. Washington, EE. UU.

Reyna-Hurtado, R., E. Rojas-Flores, y G.W.TAnner. 2009. Home range and habitat preferences of white-lipped peccaries (Tayassu pecari) in Calakmul, Campeche, Mexico. Journal of Mammalogy 90:1199-1209.

Reyna-Hurtado, R., y G. W. Tanner. 2005. Habitat preferences of ungulates in hunted and nonhunted areas in the Calakmul Forest, Campeche, Mexico. Biotropica 37:676-685.

Reyna-Hurtado, R., y G. W. Tanner. 2007. Ungulate relative abundance in hunted and nonhunted sites in Calakmul Forest (Southern Mexico). Biodiversity and Conservation 16:743-756.

Richard-Hansen, C., G. Jaouen, T. Denis, O. Brunaux, E. Marcon, y S. Guitet. 2015. Landscape patterns influence communities of medium to large-bodied vertebrates in undisturbed terra firme forests of French Guiana. Journal of Tropical Ecology 31:423-436.

Rivero, K., D. I. Rumiz, y A. B. Taber. 2005. Differential habitat use by two sympatric brocket deer species (Mazama americana and M. gouazoubira) in a seasonal Chiquitano forest of Bolivia. Mammalia 69:169-183.

Rodríguez-Mahecha, J. V., C. L. Mendoza, y S. D. Nash. 2006. Libro rojo de los mamíferos de Colombia. Conservación Internacional Colombia. Bogotá, Colombia.

Romero, M., G. Galindo, J. Otero, y D. Armenteras. 2004. Ecosistemas de la cuenca del Orinoco colombiano. Instituto de Investigaciones de Recursos Biológicos Alexander von Humboldt. Bogotá, Colombia.

Royle, J. A., y J. D. Nichols. 2003. Estimating abundance from repeated presence-absence data or point counts. Ecology 84:777-790.

SalAs, L. A. 1996. Habitat use by lowland tapirs (Tapirus terrestris L.) in the Tabaro River valley, southern Venezuela. Canadian Journal of Zoology 74:1452-1458.

Sampaio, R., A. P. Lima, W. E. Magnusson, y C. A. Peres. 2010. Long-term persistence of midsized to large-bodied mammals in Amazonian landscapes under varying contexts of forest cover. Biodiversity and Conservation 19:2421-2439.

SowLS, L. K. 1997. Javelinas and other peccaries: their biology, management, and use. Texas A\&M University Press. Texas, EE. UU.

Stanley, T. R., y J. A. Royle. 2005. Estimating site occupancy and abundance using indirect detection indices. Journal of Wildlife Management 69:874-883.

TAfur, M. P. 2010. Evaluación de la sostenibilidad de la cacería de mamíferos en la comunidad de Zancudo, Reserva nacional natural puinawai, Guainía-Colombia. Tesis de maestría. Departamento de biología. Facultad de ciencias. Universidad nacional de colombia. Bogotá, Colombia.

Tejeda-Cruz, C., E. J. Naranjo, A. D. Cuaron, H. Perales, y J. L. Cruz-Burguete. 2009. Habitat use of wild ungulates in fragmented landscapes of the Lacandon Forest, Southern Mexico. Mammalia 73:211-219.

Thorn, M., M. Green, P. W. Bateman, S. Waite, y D. M. Scott. 2011. Brown hyaenas on roads: estimating carnivore occupancy and abundance using spatially auto-correlated sign survey replicates. Biological Conservation 144:1799-1807.

Thornton, D. H., L. C. Branch, y M. E. Sunquist. 2011. The relative influence of habitat loss and fragmentation: Do tropical mammals meet the temperate paradigm? Ecological Applications 21:2324-2333.

Tobler, M. W., S. E. Carrillo-Percastegui, y G. Powell. 2009. Habitat use, activity patterns and use of mineral licks by five species of ungulate in south-eastern Peru. Journal of Tropical Ecology 25:261-270. 
Tobler, M. W., J. P. Janovec, y F. Cornejo. 2010. Frugivory and seed dispersal by the lowland tapir Tapirus terrestris in the Peruvian Amazon. Biotropica 42:215-222.

UaEspnN. 1998. Unidad Administrativa Especial del Sistema de Parques Nacionales Naturales. El sistema de Parques Nacionales Naturales de Colombia. Ministerio del Medio Ambiente. Bogotá, Colombia.

Weber, M., y S. Gonzalez. 2003. Latin American deer diversity and conservation: a review of status and distribution. Ecoscience 10:443-454.

Wilkie, D. S., E. L. Bennett, C. A. Peres, y A. A. Cunningham. 2011. The empty forest revisited. Annals of the New York Academy of Sciences 1223:120-128.

Submitted: October 15, 2015

Reviewed: December 2, 2015

Accepted: January 16, 2016

Associated editor: Rafael Reyna 